\title{
CISTO ÓSSEO ANEURISMÁTICO VERTEBRAL
}

\section{Estudo de três casos}

\author{
Benjamim Pessoa Vale', Francisco José Alencar², \\ Guilherme Brasileiro de Aguiar ${ }^{3}$, Bruno Ribeiro de Almeida ${ }^{3}$
}

\begin{abstract}
RESUMO - O cisto ósseo aneurismático é lesão hipervascularizada, benigna, localmente destrutiva pelo seu crescimento prog ressivo, de incidência maior na segunda década de vida. Acomete preferencialmente ossos longos e vértebras. Sua sintomatologia varia desde dor e edema locais, até presença de sintomas neurológicos quando de sua localização vertebral. Relatamos três casos de cisto ósseo aneurismático vertebral acometendo crianças, todas com alterações neurológicas. Os diagnósticos foram firmados através de tomografia computadorizada e/ou ressonância magnética, sendo os pacientes submetidos a ciru rgia para ressecção do tumor. Em um dos casos foi realizada a embolização arterial seletiva pré-operatória da lesão. Os três pacientes evoluíram satisfatoriamente, com melhora do quadro neurológico, demonstrando a eficácia da técnica microcirúrgica para ressecção de tumor raquimedular. Discutimos a evolução dos casos e os tratamentos existentes na atualidade.
\end{abstract}

PALAVRAS-CHAVE: tumor ósseo, cisto ósseo aneurismático, embolização arterial, cirurgia raquimedular.

\section{Vertebral aneurysmatic bone cyst: study of three cases}

ABSTRACT - Aneurysmatic bone cyst is a hypervascularized, benign lesion locally destructive by its progressive growth with greater incidence in the second decade of life. It lodges preferably in the long bones and vertebras. The clinical picture varies from pain to local edema and even neurological symptoms when in vertebral location. Three cases of vertebral aneurysmatic bone cyst occurring in childhood and all with neurologic deficit symptoms are described. Computerized tomography and/or magnetic resonance imaging confirmed the diagnosis. Patients underwent surgery to remove the tumor. In one of the cases, pre-operative selective a rterial embolization of the lesion was perf o rmed. The three patients progressed satisfactorily with neurological improvement, which demonstrated the efficiency of the microsurgical technique for the resection of the spinal tumor. The evolution of the cases and the current treatment are discussed.

KEY WORDS: bone tumor, aneurysmatic bone cyst, arterial embolization, spinal surgery.

O cisto ósseo aneurismático é lesão óssea benigna incomum, de caráter expansivo ${ }^{1-4}$, hiperv a scularizada ${ }^{4}$ e de etiologia desconhecida, ${ }^{5,6}$. Pode ocorrer em qualquer idade ${ }^{3,7}$, sendo mais freqüente em pacientes jovens ${ }^{2,4,7}$, geralmente entre 10 e 20 anos $^{7}$. Tem uma prevalência discretamente maior no sexo feminino ${ }^{3,6,8}(1: 1,04)^{6}$. Pode ocorrer em qualquer segmento do esqueleto ${ }^{7}$, sendo os sítios mais comumente acometidos os ossos longos e corpos vertebrais ${ }^{3,9}$. A localização vertebral corresponde a $10 \%$ dos casos $^{2}$, podendo corresponder até mesmo a $30 \%$ em algumas séries ${ }^{10,11}$. Sua localização vertebral pode determinar sintomatologia neurológica ${ }^{4,10,12}$, em decorrência de seu rápido crescimento e compressão de estruturas medulares e raízes nervosas. Os sintomas produzidos incluem paresias, paraplegia ${ }^{12}$, dor local ${ }^{10,13}$ e fraturas ósseas patológicas $^{10,12}$.

O presente relato tem por objetivo apresentar três casos de cisto ósseo aneurismático de localização vertebral, acometendo crianças, assim como discutir acera das diversas modalidades terapêuticas existentes na atualidade. Este estudo foi aprovado pela Comissão de Ética das instituições envolvidas.

\section{CASOS}

Caso 1 - Paciente do sexo feminino, 14 anos, queixava-se de dor lombar e fraqueza de membros inferiores há seis meses, evoluindo há 60 dias com incapacidade de deambulação. Procurou serviço de ortopedia, reali-

\footnotetext{
Hospital Infantil Lucídio Portela e Hospital São Marcos, Teresina PI, Brasil: ${ }^{1}$ Neurocirurgião com especialização em cirurgia
} neuroendovascular; ${ }^{2}$ Neurocirurgião com especialização em neurocirurgia pediátrica; ${ }^{3}$ Médico.

Recebido 31 Janeiro 2005, recebido na forma final 10 Junho 2005. Aceito 3 Agosto 2005. 


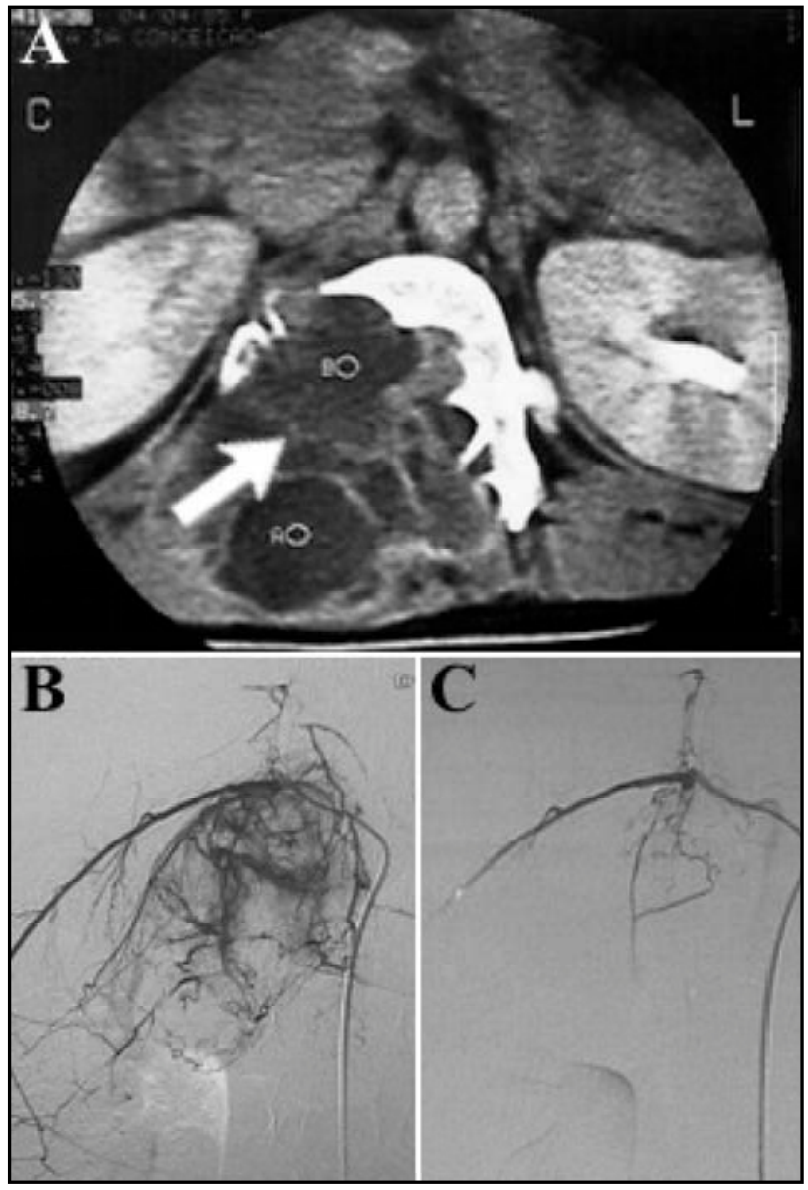

Fig 1. (A) Lesão hipervascularizada em T12, comprimindo o saco dural; (B) e (C) estudo angiográfico pré e pós-embolização, res pectivamente.

zando tomografia computadorizada (TC) de coluna vertebral, que mostrou lesão volumosa e hipervascularizada de T12, comprimindo o saco dural (Fig 1A). Foi então encaminhada ao serviço de neurociru rgia e neurorradiologia do Hospital São Marcos. O exame neurológico mostrava alterações tróficas no membros inferiores, força muscular grau I/II, reflexos profundos exaltados, além de hipoestesia com nível sensitivo em T11/T12. Apresentava ainda escaras de calcâneo. Foi realizada angiografia medular mostrando "blush" vascular opacificando-se através de T12 à direita (Fig 1B). Foi realizada embolização pré-operatória da lesão (Fig 1C) e a paciente foi então submetida à ressecção do tumor e instrumentação da coluna vertebral por via posterior. Evoluiu com meIhora do quadro neurológico e três meses após a cirurgia já apresentava exame neurológico normal.

Caso 2 - Paciente do sexo feminino, 12 anos, apresentava queixa de dor torácica com evolução há 15 dias e diminuição progressiva da força muscular nos membros inferiores há 7 dias. Evoluiu para paraplegia crural e conseqüente impossibilidade de deambular. Procurou serviço de neurocirurgia do Hospital Infantil Lucídio Por-

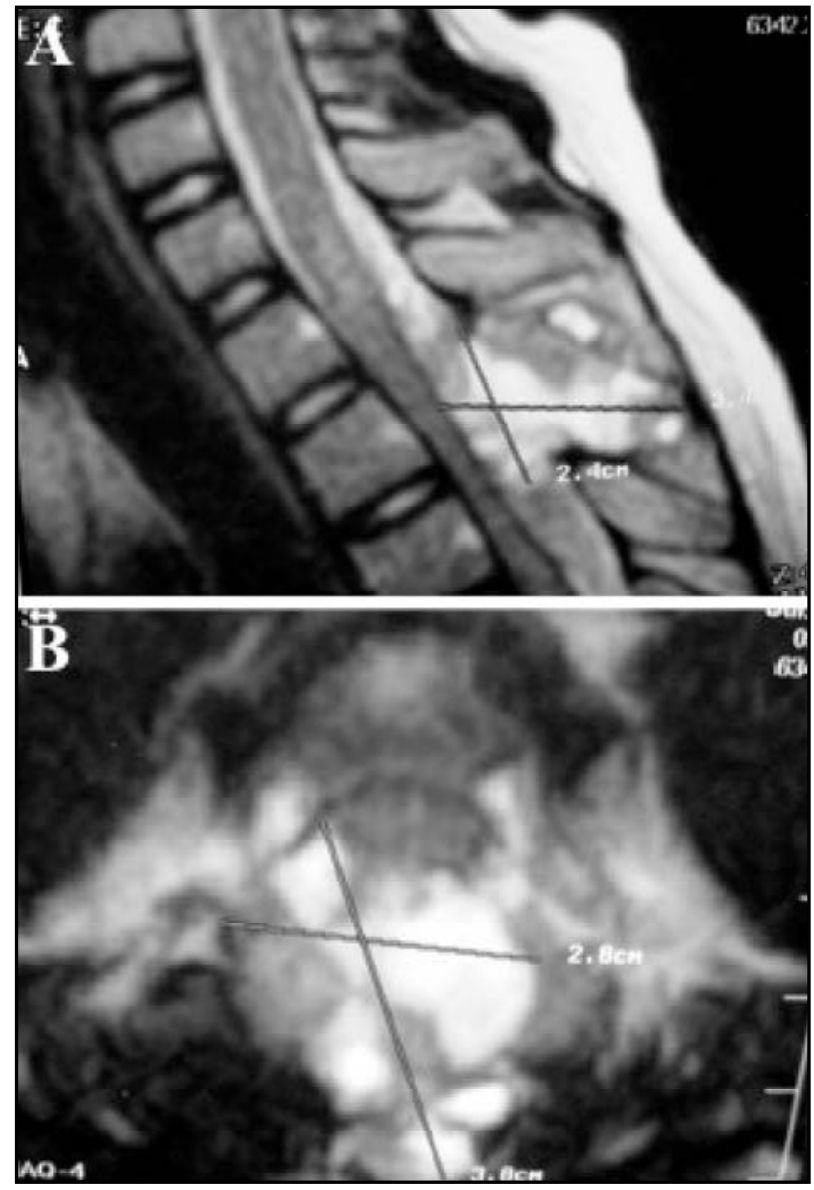

Fig 2. Lesão osteolítica em T2, em cortes longitudinal (A) e transversal (B).

tela. Ao exame neurológico demonstrava força muscular grau II nos membros inferiores e nível sensitivo em T4. A ressonância magnética (RM) demonstrou lesão expansiva hipertensa na segunda vértebra torácica, com dimensões de 2,4 X 2,8 X 3,8 cm (Fig 2). Mostrava ainda 0 arco posterior de 12 estendendo-se anteriormente para espaço epidural posterior, envolvendo parcialmente o saco dural, determinando compressão da medula espinhal. Mostrava também áreas de necrose e degeneração cística no seu interior. A paciente foi submetida a microcirurgia para tumor raquimedular. Durante $o$ ato cirúrgico apresentou sangramento importante quando da ressecção da lesão. $O$ exame histopatológico revelou tratar-se de cisto ósseo aneurismático, sendo sua re ssecção completa confirmada por exame de RM pós-operatório. Na ocasião da alta hospitalar, 12 dias após a ciru rgia, apresentava paraparesia crural, com melhora relativa do quadro neurológico. Um mês após a cirurgia, apresentava discreta melhora do quadro neurológico.

Caso 3 - Paciente de 3 anos, sexo masculino, apresentava dor lombar há mais ou menos 35 dias, tendo evoluído há 3 dias com paraparesia crural e tumoração 


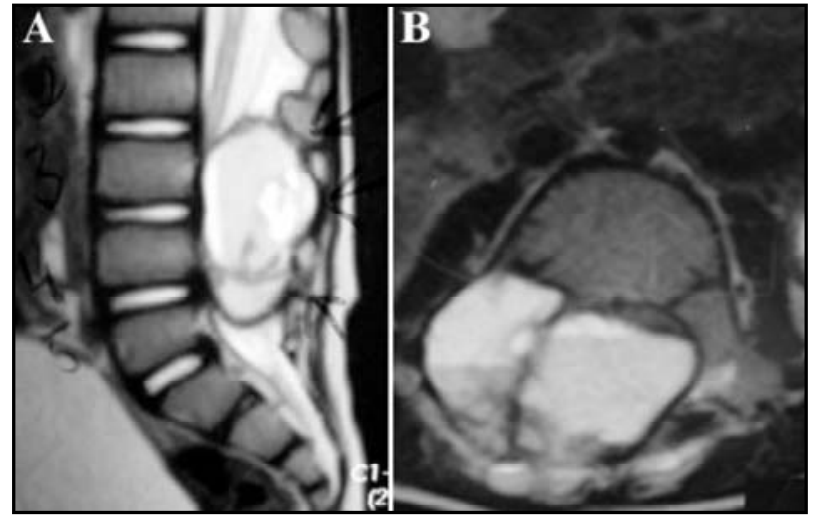

Fig 3. Lesão expansiva ao nível de L4-L5, em cortes longitudinal $(A)$ e transversal (B).

lombar. A RM demonstrou grande lesão expansiva ao nível de L4-L5, com compressão do saco dural (Fig 3). O paciente foi submetido a cirurgia para tumor raquimedular, com ressecção da lesão. Como intercorrência, durante a cirurgia ocorreu sangramento importante durante a ressecção da lesão. O exame histopatológico demonstrou tratar-se de cisto ósseo aneurismático. Recebeu alta hospitalar no $26^{\circ}$ dia pós-operatório, apresentando melhora do quadro neurológico.

\section{DISCUSSÃO}

Os tumores primários da coluna vertebral são relativamente infreqüentes quando comparados às lesões metastáticas, mieloma múltiplo e linfo$\mathrm{ma}^{14,15}$. Essas lesões secundárias sendo geralmente multifocais não despertam grandes dúvidas em relação a seu diagnóstico ${ }^{14}$. As lesões ósseas únicas, no entanto, representadas pelos tumores ósseos primários da coluna vertebral, despertam grandes considerações em relação aos possíveis diagnósticos diferenciais ${ }^{14}$. São constituídos principalmente por osteomas osteóides, osteoblastomas, tumores de células gigantes, cisto ósseo aneurismático, osteocondromas, condromas, condrosarcomas, osteosarcoma e sarcoma de Ewing ${ }^{14,15}$.

O cisto ósseo aneurismático representa uma lesão óssea benigna, osteolítica ${ }^{6}$, de apresentação solitária e de rara incidência ${ }^{1,6}$. Sua origem ainda é controversa, porém há a hipótese de que corresponde a um distúrbio hemodinâmico devido a malfo rmações venosas primárias ou secundárias dos ossos ${ }^{16,17}$. Uma lesão pré-existente pode ser identificada em até um terço dos $\operatorname{casos}^{17}$. Sua incidência é 0,14 por 100000 indivíduos ${ }^{6}$, correspondendo a $15 \%$ de todos os tumores primitivos da coluna vertebral ${ }^{2}$. Em relação ao sexo, a literatura aponta pequena predileção pelo feminino ${ }^{3,6,8}$. Os casos do presente relato ocorreram em dois pacientes do sexo feminino e um do sexo masculino, sendo suas idades menores que 20 anos, o que vem de encontro ao relatado por diversos autores ${ }^{2,6-8}$. Quando localizados na coluna vertebral, são mais freqüentes na região cervical e torácica que na localização lombar e sacral ${ }^{2}$, correspondendo aos achados em nosso estudo, em que dois casos ocorrem na coluna torácica e um caso em região lombar.

Sua sintomatologia escassa é rapidamente exacerbada quando o crescimento da lesão causa compressão de estruturas nervosas, levando ao surgimento de sintomas como dor local e irradiada ${ }^{10,11}$, p a resias, paraplegia ${ }^{12}$ e mesmo ao surgimento de fraturas patológicas ${ }^{10,12}$. O quadro sintomático leva à investigação de lesões medulares, como ocorre $u$ nos três casos relatados, nos quais os pacientes apresentavam queixas de dor, evoluindo com paresia e parestesia, até a incapacidade de deambulação.

O diagnóstico é, firmado através de exames de imagem, como a RM e a TC1,2,3,13,18. Constituem lesões osteolíticas, bem delimitadas, entre ossos e tecidos moles ${ }^{19}$. Ao exame tomográfico, o cisto ósseo aneurismático é mostrado com imagem característica, refletindo a aparência histopatológica de uma malha parcialmente cística, dividida por septos ${ }^{1}$. A RM mostra lesão circundada por uma borda completa ou incompleta de baixa intensidade de sinal ${ }^{19}$. A RM demonstra uma imagem quase homogênea de intensidade de sinal intermediária, incluindo uma área com discreto sinal parcial de baixa intensidade em T1, e uma imagem homogênea com alta intensidade de sinal que simula a aparência de bolha em T2 ${ }^{1}$. Em T1, com o uso do contraste, podese perceber o aspecto em "favo de mel" do cisto ósseo aneurismático ${ }^{1}$. Outras características dos cistos ósseos aneurismáticos incluem margens policísticas, expansão cortical, espaços císticos, bordas com áreas císticas de realce de contraste, septações inte rnas, projeções em forma de divertículo da parede do cisto ${ }^{19}$, calcificações ou ossificaçõ̃es ${ }^{20}$ e níveis fluidos ${ }^{2,19,20,21}$. A RM é o método de escolha para avaliação e diagnóstico das lesões ósseas benignas, pela sua alta sensibilidade para detectar mudanças na intensidade de sinal da medula óssea, e das áreas de tecidos moles adjacentes ${ }^{18}$, sendo assim o principal exame na avaliação do cisto ósseo aneurismático ${ }^{18}$. A RM, a TC e a angiografia foram os métodos de imagem empregados para a avaliação e diagnóstico dos pacientes em questão no presente relato. A RM mostrou processo expansivo extradural, com intensidade de sinal heterogêneo, áreas de ne- 
crose e ou degeneração cística no seu interior, determinando compressão do canal vertebral. A confirmação histológica do tipo de lesão é fundamental para o tratamento, no entanto a biópsia pré-operatória de cisto ósseo aneurismático não é indicada pelo risco de sangramento. Assim, é através dos exames de imagem que se busca o diagnóstico antes do tratamento cirúrgico ou biópsia'. No entanto, os achados de imagem são, muitas vezes, inespecíficos para o tipo histológico da lesão ${ }^{1,2}$, tornando o exame histopatológico, com o material retirado cirurgicamente, de fundamental importância para o diagnóstico final do cisto ósseo aneurismático ${ }^{1,2}$. A literatura, suporta a conduta adotada nos casos relatados, tendo em vista que o diagnóstico foi suspeitado com base nos achados de imagem, não sendo realizada biópsia pré-operatória. Após a cirurgia raquimedular, o exame histopatológico revelou tratar-se de cisto ósseo aneurismático.

A conduta adotada diante das lesões ósseas benignas ainda é muito diversificada. A indicação para o tratamento cirúrgico dessas lesões depende da sua atividade biológica, dos sintomas clínicos e de sua localização anatômica ${ }^{22}$. Lesões clinicamente silenciosas ("leave me alone lesions"), sem re alces cintilográficos, sem risco de fraturas patológicas ou transformação maligna não requerem cirurgia, somente acompanhamento ${ }^{22}$. Não foi o que ocorreu nos casos do presente relato, em que a rica sintomatologia levou à necessidade de uma rápida intervenção cirúrgica. $O$ tratamento do cisto ósseo aneurismático continua controverso ${ }^{23}$. Suas possibilidades envolvem a ressecção simples da lesão, com ou sem enxerto ósseo; a embolização da lesão, como tratamento único ou como adjuvante ao tratamento cirúrgico; o uso de radioterapia; a injeção percutânea de Ethibloc entre outros métodos $2,13,21,23-25$.

A ressecção cirúrgica do cisto ósseo aneurismático ainda é o tratamento de escolha para esse tipo de lesão ${ }^{2,23}$, promovendo um controle local e prevenindo deformidades da coluna ${ }^{23}$. Pode envolver a curetagem simples ou mesmo a realização de enxerto ósseo, em muitos casos havendo a necessidade de fixação da coluna para manutenção de sua estabilidade $e^{2,10,13,26}$. Nos casos em questão, todos foram tratados com cirurgia para ressecção da lesão, e, em somente um dos casos, houve a necessidade de fixação da coluna vertebral. Essa conduta requer algumas considerações especiais: são os casos de lesões relativamente inacessíveis, a necessidade de remoção completa da lesão para evitar recorrência, a proximidade da medula e de raízes nenosas, e a potencial instabilidade da coluna vertebral após a cirurgi $a^{23,24}$. Pode ter o sangramento excessivo durante o ato cirúrgico como complicação técnica ${ }^{4,24}$. O manejo cirúrgico é facilitado pela embolização pré-operatória do tumor hipervascularizado, possibilitando sua excisão sem perdas sanguíneas extensas intraoperatórias ${ }^{27}$. Para Boriani et al. a embolização do cisto ósseo aneurismático pode constituir até mesmo em tratamento curativo, não afetando a possibilidade de cirurgia em caso de recorrência ${ }^{10}$. Os mesmos autores preconizam o uso da embolização arterial seletiva como primeira escolha no tratamento desses tipos de lesão, salvo em casos de envolvimento neurológico, fraturas patológicas ou re co rrência, onde uma excisão cirúrgica é preconizada. No entanto, a maioria dos autores adota a ciru rgia excisional associada à embolização pré-operatória como método de escolha para o tratamento do cisto ósseo aneurismático ${ }^{24,27}$. As recomendações atuais envolvem a embolização arterial seletiva pré-operatória, a curetagem óssea excisional, o enxerto ósseo e a fixação da área afetada se houver instabilidade ${ }^{24}$. Essa foi a conduta adotada em um dos casos apresentados (primeiro caso); após a embolização arterial seletiva, foi realizada a cirurgia excisional juntamente com a fixação da coluna vertebral. Nesse caso, pôde-se confirmar a diminuição do sangramento intraoperatório, facilitando a ressecabilidade da lesão.

A injeção percutânea de Ethibloc constitui outra opção terapêutica ${ }^{21,28}$, assim como a injeção percutânea de polidocanol a $1 \%$ guiada por ultrassonografia $^{29}$. O Ethibloc é uma solução alcoólica com propriedades trombogência e fibrogênica ${ }^{28}$. Sua utilização pode aliviar a sintomatologia decorrente do cisto ósseo aneurismático, constituindo terapêutica eficaz ${ }^{21,28}$, podendo ser recomendado como tratamento de escolha para estas lesões ${ }^{21,28}$. As complicações do método incluem reação inflamatória local, de caráter transitório, e a formação de abscesso asséptico ${ }^{28}$. Esse método relativamente simples, minimamente invasivo pode tornar desnecessária uma cirurgia por cessar a expansão do cisto ósseo e induzir nova formação óssea ${ }^{28}$. Carece, no entanto, de estudos em larga escala para sua adoção como primeira escolha no tratamento de cisto ósseo aneurismático.

A radioterapia também constitui uma opção no tratamento dessas lesões ${ }^{10,13}$, podendo atuar como adjuvante, após a cirurgia, prevenindo recidivas, ou 
mesmo como tratamento único, mostrando efetividade em cisto ósseos aneurismáticos inoperáveis ${ }^{13}$.

\section{REFERÊNCIAS}

1. Asaumi J, Konouchi H, Hisatomi M, et al. MR features of aneurysmal bone cyst of the mandible and characteristics distinguishing it from other lesions. Eur J Radiol 2003;45: 108-112.

2. Dekeuwer P, Odent T, Cadilhac C, et al. Aneurysmal bone cyst of the spine in children: a 9-year follow-up of 7 cases and review of the literature. Rev Chir Orthop Reparatrice Appar Mot 2003;89:97-106.

3. Campidelli C, Di Tommaso L, Zanetti G. Aneurysmal bone cysts of the nasal cavity. Description of a case and review of the literature. Pathologica 2003;95:103-107.

4. Chen SH, Huang TJ, Hsueh S, Lee YY, Hsu RW. Unusual bleeding of aneurismal bone cyst in the upper thoracic spine. Chang Gung Med J 2002;25:183-189.

5. Otsuka T, Kobayashi M, Sekiya I, et al. Treatment of an aneurismal bone cyst of the second matatarsal using an endoscopic approach. J Foot Ankle Surg 2002;41:238-242.

6. Leithner A, Windhager R, Lang S, Haas OA, Kainberger F, Kotz R. Aneurysmal bone cyst. A population based epidemiologic study and literature review. Clin Orthop 1999;363:176-179.

7. Schulte M, Sarkar MR, Von Baer A, Schultheiss M, Suger G, Hartwig E. Therapy of aneurismal bone cyst. Unfallchirurg 2000;103:115-121.

8. Ramirez AR, Stanton RP. Aneurysmal bone cyst in 29 children. J Pediatr Orthop 2002;22:533-539.

9. Avanzi O, Joilda FG, Denzen EL, Salomão JC, Donato JP. Cisto ósseo aneurismático da coluna vertebral. Rev Bras Ortop 1996;31:103-110.

10. Boriani S, De Lure F, Campanacci L, et al. Aneurysmal bone cyst of the mobile spine: report on 41 cases. Spine 2001;26:27-35.

11. DiCaprio MR, Murphy MJ, Camp RL. Aneurysmal bone cyst of the spine with familial incidence. Spine 2000;25:1589-1592.

12. Chan MS, Wong YC, Yuen MK, Lam D. Spinal aneurismal bone cyst causing acute cord compression without vertebral collapse: CT and MRI findings. Pediatr Radiol 2002;32:601-604.

13. Feigenberg SJ, Marcus RB, Zlotecki RA, Scarborough MT, Berrey BH, Enneking WF. Megavoltage radiotherapy for aneurismal bone cyst. Int J Radiat Oncol Biol Phys 2001;49:1243-1247.

14. Leone A, Costantini A, Guglielmi G, Settecasi C, Priolo F. Primary bone tumors and pseudotumors of the lumbosacral spine. Rays 2000;25:89-103.
15. Omololu AB, Ogunbiyi JO, Ogunlade SO, Alonge TO, Adebisi A, Akang EE. Primay malignant bone tumour in a tropical African University teaching hospital. West Afr J Med 2002;21:291-293.

16. Sczendroi M, Arató G, Ezzati A, Hüttl K, Szavcsur P. Aneurysmal bone cyst: its pathogenesis based on angiographic, immunohistochemical and electron microscopic studies. Pathol Oncol Res 1998;4:277-281.

17. Kransdorf MJ, Sweet DE. Aneurysmal bone cyst: concept, controversy, clinical presentation, and imaging. Am J Roentgenol 1995;164:573-580.

18. Azouz EM. Magnetic resonance imaging of benign bone lesions: cysts and tumors. Top Magn Reson Imaging 2002;13:219-229.

19. Wörtler K, Blasius S, Hillmann A, Marx C, Brinkschmidt C, Heindel W. MR morphology of primary aneurismal bone cyst: a retrospective analysis of 38 cases. Rofo Fortschr Geb Rontgenstr Neuen Bilbgeb Verfahr 2000;172:591-596.

20. Guo Y, Hang Z. Aneurysmal bone cyst: imaging diagnosis and evaluation of therapeutic methods. Zhonghua Yi Xue Za Zhi 1996;76:676-679.

21. Falappa P, Fassari FM, Fanelli A, et al. Aneurysmal bone cyst: treatment with direct percutaneous Ethibloc injection: long-term results. Cardiovasc Intervent Radiol 2002;25:282-290.

22. Schaser KD, Bail HJ, Haas NP, Melcher I. Treatment concepts of benign bone tumors and tumor-like bone lesions. Chirurg 2002;73:1181-1190.

23. Ozaki T, Halm H, Hillmann A, Blasius S, Winkelmann W. Aneurysmal bone cyst of the spine. Arch Orthop Trauma Surg 1999;119:159-162.

24. Papagelopoulos PJ, Currier BL, Shaughnessy WJ, et al. Aneurysmal bone cyst of the spine: management and outcome. Spine 1998;23:621-628.

25. Lima JA, Mesquita GV, Pontes AV Jr, Falcão CL, Santos CPF Filho, Coelho BM. Cisto ósseo aneurismático. An Fac Med Univ Fed Pernamb 2001;46:63-66.

26. Otsuka T, Kobayashi M, Sekiya I, et al. A new treatment of aneurismal bone cyst by endoscopic curettage without bone grafting. Arthroscopy 2001;17:E28.

27. Meyer S, Reinhard H, Graf N, Kramann B, Schneider G. Arterial embolization of a secondary aneurysmatic bone cyst of the thoracic spine prior to surgical excision in a 15-year-old girl. Eur J Radiol 2002;43:79-81.

28. Garg NK, Carty H, Walsh HP, Dorgan JC, Bruce CE. Percutaneous Ethibloc injection in aneurysmal bone cyst. Skeletal Radiol 2000;29:211-216.

29. Jain R, Bandhu S, Sawhney S, Mittal R. Sonographically guided percutaneous sclerosis using $1 \%$ polidocanol in the treatment of vascular malformations. J Clin Ultrasound 2002;30:416-423. 